\title{
Estimation of the Thermodynamics of Ionic Materials
}

\section{Leslie Glasser}

\author{
Department of Chemistry, Curtin University, West Australia
}

${ }^{*}$ Corresponding author: Leslie Glasser, Professor, Department of Chemistry, Nanochemistry Research Institute, Curtin University, Perth 6845, Australia, Tel: +618 9266 3126, E-mail: L.Glasser@exchange.curtin. edu.au

\section{Citation: Leslie Glasser (2013) Estimation of the Thermodynamics of Ionic Materials. J Mater Sci Nanotechnol 1(1): e105. doi: 10.15744/2348-9812.1.e105}

\section{Received Date: June 28, 2013 Accepted Date: July 29, 2013 Published Date: August 01, 2013}

Thermodynamics provides the primary information regarding the feasibility of synthesis and stability of materials, principally through the quantities: heat capacity, $\mathrm{C}_{\mathrm{p}}$; entropy, S; enthalpy of formation, $\Delta_{\mathrm{f}} H$; the Gibbs energy of formation, $\Delta_{\mathrm{f}} \mathrm{G}$, and amplified by lattice energy, $\Delta_{\mathrm{L}} \mathrm{U}$. Values of such properties for many materials are readily available from extensive tabulations, whether printed, [1,2] online [3] or from software [4]. However, experimental thermodynamics is currently unfashionable because it is difficult and requires advanced technical skills for its performance, so that the growth of such published values falls far behind the preparation of materials. Furthermore, such tabulations can inherently not list the properties of as-yet unsynthesised and hypothesised materials. For these reasons, there is an important and permanent place for estimation methods.

Simple estimation methods for gases based on the extensive database of known values have been established. These consist of the methods of group contributions [3] together with statistical mechanics, relying on the independence of the molecules from one another. The problem for condensed phases is more intractable because account must also be taken of the interactions between species. Molecular mechanics and quantum mechanics are available computational tools for estimation, but both are somewhat problematic for general use, because of the necessity to establish appropriate force fields (for the former) and their general complexity in operation.

However, it has been recognised over recent years that the properties of ionic materials are rather simple because they are dominated by the long-range coulombic (charge) and short-range repulsive forces present, with relatively minor contributions from other interactions. This implies that detailed consideration of the structures of ionic materials is unnecessary, so that simple relations may be expected between ionic materials, based solely on their charge identities and the closeness of packing of their charges - that is, on their formula volumes. This realisation has resulted in the preparation of tables of single-ion values for volume, [5] heat capacity, [6] entropy, [7] enthalpies of formation and Gibbs energies of formation [8] which are simply additive, yet yield reliable results. Furthermore, it has been demonstrated for numerous ionic solids and even ionic liquids that there are excellent linear correlations between formula volume and these same thermodynamic properties; $[9,10]$ we have named estimations by these means as "Volume-Based Thermodynamics" (VBT). This is a particularly valuable resource since the basic variable of formula volume is the most readily available thermodynamic quantity: through measurement of density; from structure by $\mathrm{x}$-ray diffraction; through simple differences among related materials; or even simple estimation by single-ion addition. This additivity can even be taken further, through various "difference rules" [11-13] between related species and the "Simple Salt Approximation" (SSA), [14] whereby the properties of simple constituent salts are summed to yield the properties of a more complex salt (for example: $\mathrm{MgO} \bullet \mathrm{Al}_{2} \mathrm{O}_{3} \rightarrow \mathrm{MgAl}_{2} \mathrm{O}_{4}$ : spinel). These methods are sufficiently simple to be used (with care and consideration) to yield reliable results even in unpracticed hands, and as a check on not-always reliable published values.

\section{References}

1. Lide DR (2007) CRC Handbook of Chemistry and Physics. (87 $7^{\text {th }}$ edn), CRC Press, Boca Raton, USA.

2. Chase MW (1998) NIST-JANAF Thermochemical Tables. American Chemical Society, Washington DC, USA.

3. Linstrom PJ; Mallard WG (2011) NIST Standard reference database number 69. NIST Chemistry WebBook.

4. (2009) Outotec Research Oy HSC Chemistry 7. Outotec, Finland.

5. Glasser L, Jenkins HD (2008 Internally consistent ion volumes and their application in volume-based thermodynamics. Inorg Chem 47: 6195-6202.

6. Glasser L, Jenkins HD (2012) Single-ion heat capacities, C (p) (298) ion, of solids: with a novel route to heat-capacity estimation of complex anions. Inorg Chem 51: 6360-6366.

7. Glasser L, Jenkins HD (2009) Single-ion entropies, S(ion)(o), of solids--a route to standard entropy estimation. Inorg Chem 48: 7408-7412.

8. Glasser L (2013) Single-ion values for ionic solids of both formation enthalpies, $\Delta$ (f) H (298) (ion), and Gibbs formation energies, $\Delta$ (f) G (298) (ion). Inorg Chem 52: 992-998.

9. Glasser L, Jenkins HDB (2011) Volume-based thermodynamics: a prescription for its application and usage in approximation and prediction of thermodynamic data. J Chem Eng Data 56: 874-880. 
10. Glasser L, Jenkins HDB (2000) Lattice energies and unit cell volumes of complex ionic solids. J Am Chem Soc 122: 632-638.

11. Jenkins HDB, Glasser L (2010) Thermodynamic difference rules: a prescription for their application and usage to approximate thermodynamic data. J Chem Eng Data 55: 4231-4238.

12. Jenkins HDB, Glasser L, Liebman JF (2010) The Thermodynamic Hydrate Difference Rule (HDR) Applied to Salts of Carbon-Containing Oxyacid Salts and Their Hydrates: Materials at the Inorganic-Organic Interface. J Chem Eng Data 55: 4369-4371.
13. Glasser L, Jenkins HD (2007) The thermodynamic solvate difference rule: solvation parameters and their use in interpretation of the role of bound solvent in condensed-phase solvates. Inorg Chem 46: 9768-9778.

14. Yoder CH, Flora NJ (2005) Geochemical applications of the simple salt approximation to the lattice energies of complex materials. Am Mineral 90: 488-496.

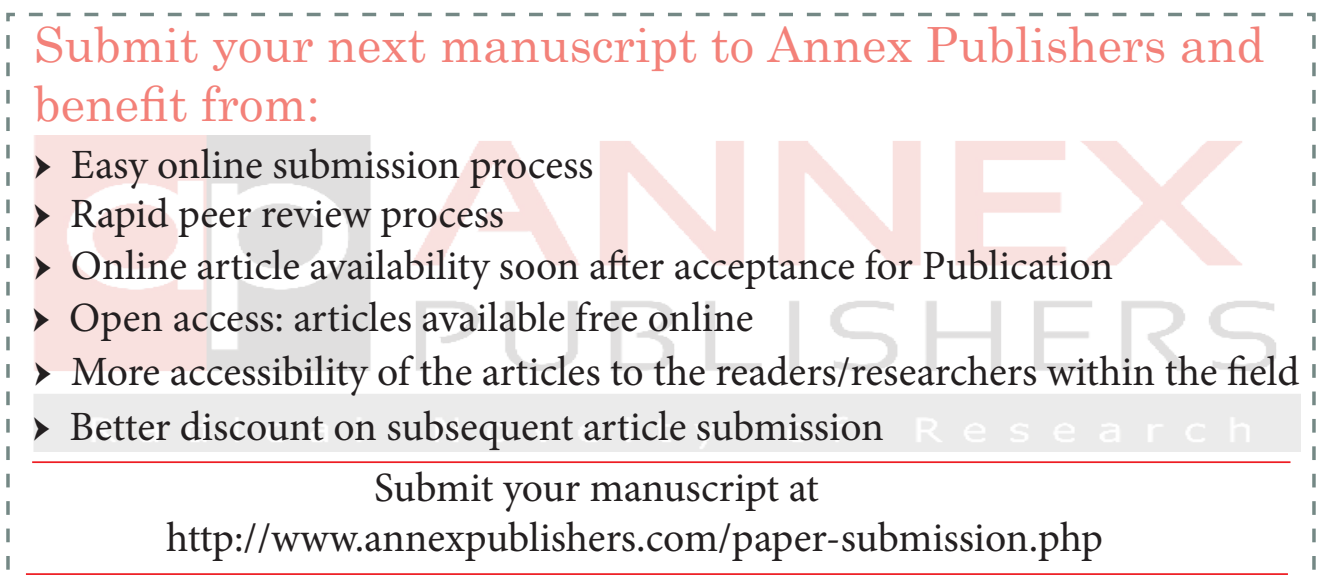

